\title{
Rheuma und Ernährung
}

\section{Was kann empfohlen werden?}

\begin{abstract}
A. Michalsen ${ }^{1,2}$
${ }^{1}$ Charité - Universitätsmedizin Berlin, Stiftungsprofessur für klinische Naturheilkunde; Institut für Sozialmedizin, Epidemiologie und Gesundheitsökonomie; ${ }^{2}$ Immanuel Krankenhaus Berlin, Abteilung und Zentrum für Naturheilkunde, Klinik für Innere Medizin
\end{abstract}

\section{Schlüsselwörter \\ Rheuma, Ernährungstherapie, Fasten, Diät}

\section{Zusammenfassung}

Viele Patienten mit rheumatischen Erkrankungen fragen nach den Möglichkeiten, ihre Erkrankung über die Ernährung zu beeinflussen. In Erhebungen und epidemiologischen Studien werden immer wieder Ernährungsfaktoren beschrieben, die mit dem Auftreten rheumatischer Erkrankungen bzw. von Schüben verbunden sind, allerdings kann kein einheitliches Ernährungsmuster beschrieben werden. Wie allgemein im nichtpharmakologischen Therapiebereich stehen nur in begrenztem Umfang Daten aus randomisierten Studien zur Evidenzbewertung zur Verfügung. Für die rheumatoide Arthritis (RA) belegen zwei randomisierte Studien die Wirksamkeit der mediterranen Ernährung. Für die vegetarischen Kostformen weisen v. a. experimentelle Daten auf grundsätzlich deutliche antientzündliche Effekte und klinische Studien auf günstige klinische Wirkungen bei kardiovaskulären Erkrankungen hin. Die Wirksamkeit glutenfreier Diäten bei RA ist fraglich und glutenfreie Diäten sollten nur bei Zöliakie oder starken Hinweisen auf eine Nicht-Zölia- kie Glutensensitivität (NCGS) empfohlen werden. Mehrere Studien und eine systematische Übersicht belegen die Wirksamkeit eines sieben- bis zehntätigen Fastens auf die Symptome und Funktion bei RA. Folgt auf das Fasten eine vegane und vegetarische Ernährung, sind nachhaltige Effekte von bis zu einem Jahr dokumentiert. Der mögliche Nutzen verschiedener spezifischer Nahrungsmittel und Nahrungsergänzungen wie grüner Tee, Granatapfel, Ingwer, Leinöl, Gelbwurz ist klinisch derzeit nicht ausreichend belegt, aber v. a. für Gelbwurz (Curcuma) vielversprechend. Neuere klinisch-experimentelle Daten belegen den Einfluss des Darm-Mikrobioms auf die Entstehung der Erkrankung. Allerdings sind bislang spezifische probiotische Therapieansätze nicht ausreichend durch Daten aus klinischen Studien gestützt.

\section{Keywords}

Rheumatic disease, nutritional therapy, fasting, diet

Rheumatic disease and nutrition

Current recommendations

arthritis + rheuma 2018; 38: 356-362

\section{Summary}

There is an increasing interest for nutritional therapies in patients with rheumatic diseases. Patients and some smaller surveys are frequently reporting causal associations of the beginning of disease or symptomatic flareups. However, there is no homogenous profile of such nutritional factors. As generally with non-pharmacological treatments evidence from randomized controlled trials is scarce. Two randomized studies have proven the effectiveness of the traditional Mediterranean diet in patients with rheumatoid arthritis (RA). For vegetarian diets, experimental evidence shows significant anti-inflammatory effects while clinical studies underline its value in the prevention and treatment of cardiovascular and metabolic diseases. The value of gluten-free elimination diets remains unclear. Several studies and a systematic review point to the effectiveness of periods of 7 to 10 days of modified fasting on symptoms and function in RA. Fasting followed by a vegan and lactovegetarian diet leads to a prolonged beneficial effect over one year. The potential benefit of distinct nutrients and nutritional supplements as green tea, pomegranate, ginger, curcuma, linseed oil is not proved sufficiently by clinical trials so far, however data for curcuma/turmeric are promising. Preliminary data show associations between alterations of the gut microbiome and rheumatic diseases. So far there is no sufficient evidence supporting the recommendation of specific probiotic treatments or prebiotic diets in the treatment of rheumatic diseases.
Eine Vielzahl von Patienten mit rheumatoider Arthritis aber auch Spondylarthropathien und Kollagenosen berichten in der Anamnese über ihre Erfahrung, dass Ernährung bzw. gewisse Ernährungsfaktoren die Krankheit positiv oder negativ beeinflussen und Krankheitsschübe auslösen können. Darüber hinaus haben Patienten mit rheumatischen Erkrankungen ein erhöhtes Risiko für das metabolische Syn- drom und kardiovaskuläre Erkrankungen (1).

Auch die nicht seltene rheumatische Kachexie mit entzündungsbedingter Sarkopenie und erhöhtem viszeralem Fett 
weist auf mögliche ernährungstherapeutische Therapieansätze (2). Insgesamt besteht bei Patienten mit rheumatischen Erkrankungen und mit chronischen Schmerzsyndromen, einschließlich bei Familien mit rheumakranken Kindern bzw. Jugendlichen, ein großes Interesse und wachsende Nachfrage zu den Möglichkeiten der Ernährungstherapie. In der klinischen Naturheilkunde und ihren stationären und ambulanten Einrichtungen wird der Ernährung und weiteren Faktoren des gesundheitsfördernden Lebensstils traditionell ein großer Stellenwert in der Beratung und Therapie beigemessen.

Der jahrzehntelangen klinischen Erfahrung steht jedoch eine vergleichsweise nur begrenzte Datenlage aus randomisierten Studien gegenüber, sodass weder für positive noch negative Aussagen derzeit eine zufriedenstellende Evidenz besteht. Im Folgenden werden die wichtigsten für rheumatische Erkrankungen relevanten ernährungstherapeutischen Methoden beschrieben und die klinische Erfahrung und Evidenz bei Erwachsenen dargestellt, da entsprechende Daten für rheumakranke Kinder und Jugendliche fehlen. Wünschenswert ist, dass in Zukunft mehr Forschungsförderung in diesem Bereich zur Verfügung gestellt wird. Dies würde auch der Tatsache Rechnung tragen, dass das Interesse an Ernährungs- und Essensthemen in der Bevölkerung und bei Patienten mit chronischen Erkrankungen und deren Familien seit Jahren stetig zunimmt und aktuelle zentrale Bereiche der experimentellen Grundlagenwissenschaft zunehmend auf eine bedeutsame Rolle der Ernährung hinweisen (u.a. intestinale Mikrobiota, Epigenetik).

\section{Ernährung als ursächlicher Faktor bei Rheuma}

Bereits in frühen Befragungsstudien von Darlington wurde dokumentiert, dass $\mathrm{Pa}$ tienten mit rheumatischen Erkrankungen häufig über eine Zunahme ihrer Gelenkbeschwerden nach dem Verzehr spezieller Nahrungsmittel berichten (3). Für die rheumatoide Arthritis waren dies insbesondere Fleisch jeder Art, Milch, Eier, Weizenprodukte sowie Mais, bei Arthrose
Fleisch, Zitrusfrüchte, Süßwaren und Schokolade sowie Alkohol. Darauf aufbauende Eliminationsdiäten haben allerdings in Studien, u.a. der Arbeitsgruppe von Darlington, keine schlüssigen und für die Ernährungsberatung konkreten Ergebnisse erbracht.

Die größte Datenauswertung zur Ernährung und rheumatoider Arthritis liefert eine aktuelle Analyse der Nurses Health Studie und der NHSII-Studie mit insgesamt über 170000 Frauen, die über 22 Jahre beobachtet wurden (4). Die in diesem Zeitraum aufgetretenen 1185 Erstdiagnosen einer RA wurden mit dem Ernährungsverhalten assoziiert. Insbesondere wurde 18 Ernährungsfaktoren als pro- oder antiinflammatoisch skaliert (u.a. Fleisch, Wurst zuckerhaltige Getränke $=$ proinflam matorisch) und in einem Gesamt-Score addiert. Für die Gesamtpopulation ergab sich kein statistisch signifikanter Zusammenhang. Für Frauen mit Krankheitsbeginn $<55$ Jahren und seropositiver RA zeigte sich hingegen ein starker Zusammenhang mit Erhöhung des Krankheitsrisikos um $38 \%$ bei Ernährung mit hohem Index. Bei der weiteren statistischen Adjustierung erwies sich dieser Zusammenhang partial abhängig vom Vorliegen von Übergewicht und Adipositas.

\section{Klinische Studien zu Ernährungsformen}

Die Wirksamkeit von Ernährungsformen bei rheumatischen Erkrankungen wurde in wenigen klinischen randomisiert kontrollierten Studien evaluiert. Relativ gut untersucht ist v.a. die Wirksamkeit der mediterranen Ernährung bei rheumatoider Arthritis.

\section{Traditionelle Mittelmeerkost}

Die traditionelle Mittelmeerkost ist reich an Vegetabilien, Getreide, Obst, Hülsenfrüchten, Samen und Nüssen, setzt überwiegend Olivenöl als Speiseöl ein und enthält nur geringe Mengen Fleisch, Milchprodukte sowie in küstennahen Regionen gelegentlich Fisch. Skoldstam und Mitarbeiter untersuchten die Wirkungen der mediterranen Kost bei 56 Patienten mit RA in einer randomisiert kontrollierten Studie (5). Nach zwölf Wochen resultierte eine signifikante Differenz zugunsten der mediterranen Diät in Bezug auf die Schmerzstärke und tendenziell für die Funktion. Patienten in der sich mediterran ernährenden Gruppe hatten eine signifikant geringere Krankheitsaktivität im DAS28 und eine verbesserte Lebensqualität. Eine zweite, von McKellar et al. publizierte Studie wurde bei 130 Patienten in Schottland durchgeführt (6). Es zeigte sich ebenfalls ein signifikanter Rückgang der Schmerz-Scores in der Interventionsgruppe nach drei und sechs Monaten. Darüber hinaus zeigte sich eine Verbesserung der Funktion im HAQScore und eine Verbesserung der Morgensteifigkeit nach sechs Monaten durch eine mediterrane Kost. Da die Wirksamkeit der mediterranen pflanzenbasierten Kost für die Prävention und Therapie der KHK, des Bluthochdrucks, der Demenz und des Diabetes mellitus mit starker Evidenz unterlegt ist (7-8), sollte Patienten mit einer rheumatoiden Arthritis, die auch unter erhöhter kardialer Morbidität und Mortalität leiden, eine mediterrane Ernährung als mögliche gesunde Basisernährung empfohlen werden.

\section{Vegetarische Ernährung}

Der Nutzen vegetarischer Kostformen ist für Bluthochdruck, Diabetes mellitus sowie die Prävention und Therapie koronarer Erkrankungen belegt (9-12). Für die rheumatoide Arthritis besteht Evidenz aus einer randomisierten Studie, in der initial eine sieben- bis zehntägige Fastenperiode durchgeführt wurde. Darauf erfolgt über drei Monate eine vegane und glutenfreie Ernährung, gefolgt von einer stufenweisen Wiedereinführung einer laktovegetabilen Vollkost.

In den Ergebnissen zeigte sich eine deutliche und signifikante symptomatische Verbesserung nach eine Jahr gegenüber der Kontrollgruppe (13). Unklar bleibt, welchen Anteil am Gesamteffekt die initiale Fastenperiode und die nachfolgende vegetarische Ernährung jeweils hatten. Tierexperimentelle Daten der letzten Jahre belegen aber zunehmend, dass schwefelhaltige Aminosäuren tierischer Herkunft, insbesondere Methionin, eine starke proinflam- 
matorische Wirkung aufweisen (14-16). Seit langem ist zudem bekannt, dass Arachidonsäure aus Fleisch- und Milchprodukten über die entsprechende Eicosanoidsynthese entzündungsfördernd wirken. Aufgrund dieser Datenlage kann Patienten mir rheumatischen Erkrankungen eine mediterrane oder asiatische vegetarische oder vollwertige vegane Ernährung empfohlen werden. Bei veganen Ernährungsformen ist auf die Supplementierung von Vitamin B12 zu achten. Weitere kritische Nährstoffe werden bei einer vollwertig durchgeführten veganen Ernährung ausreichend aufgenommen. Hier sollte aber eine entsprechend ausgewiesene Ernährungsberatung veranlasst werden.

Nicht zu empfehlen sind kohlenhydratreduzierte Ernährungsformen (Low Carb) wie die Atkins-Diät, die zwar kurzfristig zu effektiver Gewichtsabnahme führen, aber langfristig mit geringer Compliance und einer Verschlechterung des kardiovaskulären Risikoprofils assoziiert sind.

\section{Therapeutisches Fasten („Heilfas- ten") und kalorische Restriktion}

In den integrativen Therapiekonzepten der naturheilkundlichen Therapie rheumatischer Erkrankungen spielt das therapeutische Fasten („Heilfasten“) eine herausragende Rolle. Die Bereitschaft, sich auf diese auch Selbstdisziplin abverlangende Therapie einzulassen, ist in der Regel bei chronisch Erkrankten hoch. Die Lebenszeitprävalenz einer Vorerfahrung mit Fasten unabhängig von Motiv und Methode schwankt in der erwachsenen Bevölkerung je nach Stichprobe und Präzisierung der Frage, insbesondere bezüglich der Mindestfastendauer, zwischen 14 und 50\%.

Fasten, definiert als der freiwillige und zeitlich definierte Verzicht auf feste Nahrung und Genussmittel, hat weltweit eine umfassende ethnomedizinische und kulturelle Tradition. In der menschlichen Evolution dürfte die körperliche Auseinandersetzung mit jahreszeitlich bedingten Phasen eines mangelnden Nahrungsangebotes die Regel gewesen sein. Die Fähigkeit, sich diesen Schwankungen durch Depotbildungen und deren Mobilisierung anzupassen, war Bedingung für das menschliche Überleben. Sie bildet die physiologische Grundlage für die Fähigkeit, für eine begrenzte Zeit den Bedarf an Mikro- und Makronährstoffen bei ausbleibender oder nur minimaler Nahrungsaufnahme ohne gesundheitliche Nachteile aus körpereigenen Reserven zu decken. Darüber hinaus kann aufgrund einer Vielzahl von inzwischen vorliegenden wissenschaftlichen Daten hypothetisiert werden, dass periodische Fastenzeiten zusätzliche physiologisch-therapeutische Wirkungen haben. Diese umfassen metabolische, neuroendokrine, immunologische und psychologische Effekte. Aus der biologischen Fähigkeit entwickelten sich weltweit Fastenkulturen mit religiös-spiritueller als auch medizinisch-therapeutischer Zielsetzung. In der Entwicklung seit dem 20. Jahrhundert integrierte sich die Fastentherapie bzw. das therapeutische Fasten oder „Heilfasten“ über das Fachgebiet der Naturheilkunde in die Medizin.

Als Fastenformen haben sich im deutschsprachigen Raum vor allem die modifizierten Methoden nach Otto Buchinger (Saftfasten, Erstveröffentlichung 1935) und nach F.X. Mayr (1921) etabliert. Die am häufigsten angewendete Fastenmethode ist dabei das Heilfasten nach Otto Buchinger (1878-1966). Der Internist und ehemalige Marinearzt eröffnete 1920 die erste Heilfastenklinik in Deutschland, nachdem er durch das Heilfasten eine ausgeprägte Besserung seiner retrospektiv nicht mehr ganz zu spezifizierenden rheumatischen Erkrankung erfahren hatte. Bei dieser Form des Fastens werden kleine Mengen Obst oder Gemüsesaft (täglich zweimal 150 bis $200 \mathrm{ml}$ ) und eine gefilterte Gemüsebrühe verzehrt. Die Trinkmenge ist frei. Eine gewisse Kalorienzufuhr erfolgt, um Eiweißkatabolismus vorzubeugen. Das Saftfasten nach Buchinger kann als sehr verträglich eingestuft werden. Registerdaten mit bis zu 20000 Patienten zeigen bei Fastendauern von 5-14 Tagen keine ersthaften unerwünschten Wirkungen, wenn Indikationen und Kontraindikationen beobachtet werden.

\section{Kalorische Restriktion und Fasten- therapie im Kontext neuerer Grund- lagenforschung}

Tierexperimentell ist seit längerem bekannt, dass eine nutritive Kalorienrestrikti- on von etwa $30 \%$ des täglichen Nahrungsenergieangebotes oder, alternativ, intermittierendes Fasten zu einer Lebensverlängerung und Reduktion inflammatorischer, degenerativer und altersabhängiger Erkrankungen bei allen Tierspezies führt (15-17). Bemerkenswert ist dabei, dass nicht nur häufige altersbedingte Erkrankungen, wie etwa Diabetes, Arteriosklerose, Krebs oder Demenz, später auftreten, sondern sich die maximale Lebensdauer bzw. Lebensspanne der Spezies um etwa 30 \% verlängert. Damit stellen intermittierendes Fasten und kalorische Restriktion die bislang einzig bekannten Methoden dar, die mit einer Verlängerung der „Healthy Life Span" verbunden sind (18). Da eine dauerhafte kalorische Restriktion nur schwer einzuhalten ist, hat sich sowohl experimentell als auch in der klinischen Translation der Focus auf die günstigen medizinischen Wirkungen des intermittierenden oder prolongierten periodischen Fastens gelegt. Beispielsweise zeigen Mäuse und Ratten, die ein intermittierendes Fastenschema erhalten (24 Stunden Essen ad libitum und 24 Stunden Fasten im Wechsel) eine 30-35\%ige längere Lebenserwartung sowie selteneres Auftreten von rheumatischen Entzündungen, aber auch von Diabetes, Niereninsuffizienz, Herzinsuffizienz, und neurodegenerativen Erkrankungen (15).

Zahlreiche Grundlagenstudien konnten wesentliche Mechanismen der antientzündlichen Wirkung des Fastens identifizieren $(15,18,19)$. Hierbei führt die intensive kalorische Restriktion $\mathrm{zu}$ vermehrter Signaltransduktion entzündungshemmender Proteine und der Inhibition von mTOR-Signalproteinen, zur Absenkung der Insulin- und IGF-1-Konzentration und zu vermehrter Autophagie. Weiter scheinen fasteninduzierte Veränderungen der intestinalen Mikrobiota sowie die Leptindepletion mit konsekutiver T2-Lymphoyztensuppression eine maßgebliche Rolle zu spielen. Ein weiterer Mechanismus zentriert sich auf die fasteninduzierte Bildung des Ketonkörper $\beta$-Hydroxybuttersäure (BHB), was wiederum die Expression proinflammatorischer Gene inhibiert (19). 


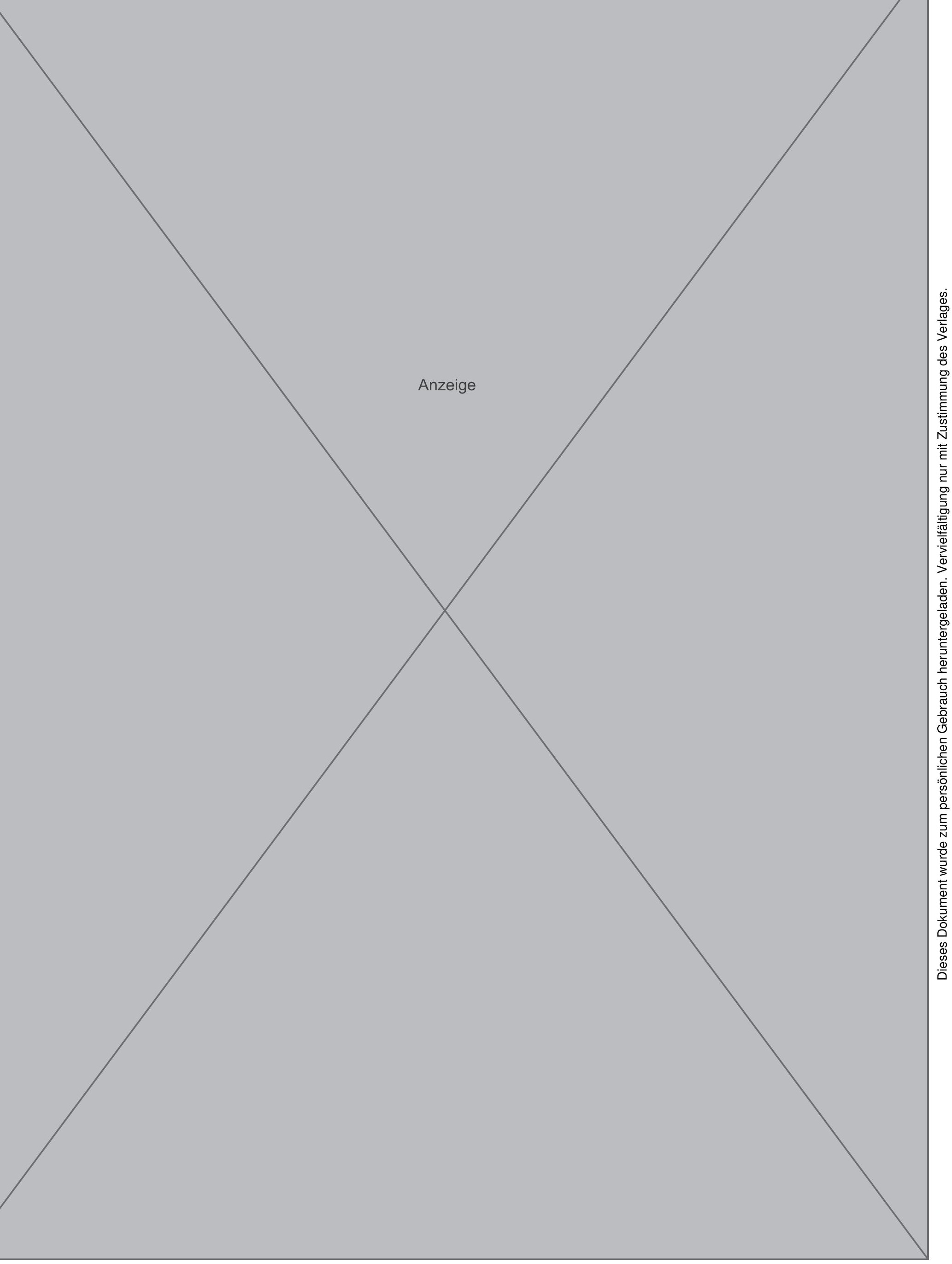




\section{Klinische Evidenz zum Heilfasten}

Die klinische humane Erforschung des Fastens begann bereits in den 80er-Jahren des 20. Jahrhunderts vor allem in Skandinavien. Dort wurden vier randomisierte Studien zum Fasten bei rheumatoider Arthritis publiziert, die später auch zu einem systematischen Review führten, der eine langanhaltende, vor allem schmerzlindernde Wirkung beschreibt, aber weitere Studien fordert (20). In der schon erwähnten Studie von Kjeldsen-Kragh et al. sollten die Patienten nach dem Fasten eine dreimonatige vegane und glutenfreie Ernährung praktizieren, ehe sie schrittweise tierische und glutenhaltige Lebensmittel unter strenger Beachtung von sogenannten Triggereffekten wieder einführten (s. o.) (13). Aus der einjährigen Nachbeobachtungsphase wurden dauerhaft günstige Effekte beschrieben, die den maximalen Effekten der DMARD der Vor-Biologika-Ära entsprachen. Im Vergleich zu den Effekten heutiger Therapie unter Einschluss der Biologika sind die Effekte aber deutlich geringer. Insgesamt können auch, bedingt durch die kleine Fallzahl der damaligen Studien und die limitierte Beobachtungsdauer, keine Aussagen über die Wirkung des Fastens auf die radiologische Krankheitsprogression gemacht werden. Vor diesem Hintergrund wird Patienten mit rheumatoider Arthritis in den führenden naturheilkundlichen Einrichtungen explizit das Fasten nur als komplementäre bzw. ergänzende Therapie zur DMARD-Medikation vermittelt und nicht als Alternative (auch wenn Patienten oft diesen Wunsch äußern).

Zur Erzielung eines signifikanten schmerzlindernden und entzündungshemmenden Effektes ist eine Mindestfastendauer von sieben bis zehn Tagen notwendig. Praktisch-klinisch ist die Fastenmethode bei gegebener Erfahrung leicht in komplexstationäre Behandlungsabläufe integrierbar. Am Zentrum für Naturheilkunde am Immanuel Krankenhaus Berlin wird die Fastentherapie bei etwa $70 \%$ aller Patienten mit RA, Spondylarthropathien (SpA) oder Kollagenosen durchgeführt und zeigt sich in der Anwendung sicher bei guter Compliance. Insbesondere Hunger wird bei Anwendung einer richtigen Fastentechnik nur selten empfunden. Viele Pa- tienten wiederholen die Fastentherapie in Abständen von sechs bis zwölf Monaten, um den Therapieeffekt zu reproduzieren. Kontraindikationen des Fastens, wie anamnestisch vorliegende Essstörungen, weight cycling und Malnutrition, sind zu beachten. In Einzelfällen kann das Fasten auch ein Stressor z. B. für Schleimhäute des Magen-Darm-Traktes sein, was zum sorgfältigen Umgang mit nichtsteroidalen Antirheumatika, Coxiben und Kortikosteroiden veranlasst. Auch Gichtanfälle und Gallenkoliken können als unerwünschte Wirkungen vorkommen und bilden bei entsprechender Anamnese eine relative Kontraindikation.

Mehrere laufende internationale Studien untersuchen derzeit verschieden Techniken des Fastens und ihre Wirkungen bei RA.

\section{Eliminationsdiäten - Gluten und andere Weizenallergene}

Diese sind in den letzten Jahren zunehmend Thema bei Rheumakranken. Eine Ausgangsprämisse ist die bei vielen Autoimmunerkrankungen auf gemeinsame Ursachen hinweisende erhöhte Überlappungswahrscheinlichkeit, d.h. die Wahrscheinlichkeit für die Manifestation zweier oder auch mehr als zwei Erkrankungen in einer Person ist deutlich höher als die meist extrem niedrigen Produkte ihrer Einzelwahrscheinlichkeiten. Dies gilt auch für RA und Zöliakie. In der Praxis ist es leicht erfahrbar, z. B. für Hashimoto-Thyreoiditis und RA. Damit ist natürlich a priori nicht gesagt, dass nur eine Therapie gleichzeitig für mehrere Autoimmunerkrankungen effektiv sein kann. Zöliakie kann zwar auch Gelenkbeschwerden bereiten, die auf glutenfreie Ernährung ansprechen. Gesichert Erkrankte stellen aber nur einen winzigen Bruchteil unter den RA-Patienten dar. Es sind aber in den letzten beiden Dekaden Konzepte für das wesentlich häufigere Auftreten von weniger klar erkennbaren Varianten der Sprue entstanden, insbesondere der „silent sprue“. In diesem Rahmen ist eine glutenfreie Ernährung auch bei RA propagiert worden. Unter Hinweis auf den experimentellen Charakter kann eine individuelle Austestung mittels glutenfreier Ernährung, die durch das vielfältige Produkt- angebot immer leichter praktizierbar wird, für einen Zeitraum von maximal zwei Monaten probatorisch in Einzelfällen empfohlen werden, in denen sich dann aber deutliche Besserungen des Verlaufs der RA zeigen sollten. Ob eine Nicht-Zöliakie Glutensensitivität (NCGS) auch relevante ungünstige Wirkungen auf die RA haben kann, ist nicht bekannt. Diese lässt sich durch eine jeweils placebokontrollierte Elimination und Reexposition sichern, bestätigt sich dann meist nur bei einer kleinen Untergruppe der Patienten mit vermuteter Glutenunverträglichkeit. Die Pathophysiologie der NCGS ist nicht eindeutig geklärt, aber vermutlich sind neben Gluten auch andere Getreidebestandteile wie ATI (Amylase-Trypsin-Inhibitoren) und die auch beim Reizdarmsyndrom relevanten FODMAPs (fermentierbare Oligo-, Diund Monosaccharide und Polyole) für Symptome verantwortlich. Eine glutenfreie Ernährung kann hier leichte günstige Effekte zeigen. Aufgrund des Aufwandes und auch des Risikos gesundheitlicher Nachteile durch eine dauerhafte Gluteneliminationsdiät ist eine graduierte Reexposition mit Ermittlung individueller Toleranzgrenzen eine sinnvolle Alternative für viele Patienten.

Komplexe sehr umfangreiche Eliminationen wurden einmal in einer randomisierten Studie getestet (21). Die Erfolge in der Interventions- im Vergleich zur Kontrollgruppe waren relativ groß. Allerdings bestand die Intervention in einer sowohl glutenfreien wie auch veganen Kost, was allerdings eine nur eingeschränkt alltagskompatibele Ernährung mit sich bringt.

Insgesamt ist eine gründliche ärztliche Aufklärung über die in der Regel nur geringen Effekte von Eliminationen auf den Verlauf der RA wichtig, um unrealistische Erwartungen an oft auch selbstheroisierende Ernährungspraktiken zu begegnen. Der mit solchen Fragen öfter beschäftigte Arzt wird immer wieder Patienten begegnen, die hier den Erfolg solcher selbstgewählten Maßnahmen überbewerten.

\section{Nahrungsergänzungen}

Im Bereich der Nahrungsergänzung werden in der Naturheilkunde häufig Curcuma (Gelbwurz), Ingwer, Grüner Tee, Lein- 
samen und Walnüsse (Alpha-Linolensäure, pflanzliche Omega-3-Fettsäuren) sowie Granatapfel bei rheumatischen Erkrankungen empfohlen. Für diese und insbesondere für Gelbwurz bzw. Präparate mit dem Hauptinhaltsstoff der Gelbwurz, Curcumin, liegen eine Vielzahl experimenteller Studien und inzwischen auch erste kleinere klinische Studien vor, die auf eine antirheumatische Wirkung hinwiesen (22-32). Experimentelle Studien ergaben zudem Hinweise auf antirheumatische Wirkungen von Heidelbeeren, Erdbeeren und Quercetin-haltigen Nahrungsmitteln (Zwiebel, Knoblauch). Einige experimentelle Studien weisen auch auf antiinflammatorische Effekte von Olivenöl „extra vergine“ hin. Hierbei sind wohl vor allem die darin enthaltenen Polyphenole das wirksame Agens und weniger die einfach ungesättigten Fette. Für diese Nahrungsergänzungen gibt es jeweils zwar vielversprechende experimentelle Daten, aber noch keine ausreichende Evidenz aus klinischen Studien.

\section{Mikrobiom}

Wissenschaftlich derzeit von großem Interesse ist die potenzielle Immunmodulation durch gezielte Beeinflussung der intestinalen Mikrobiota durch Pro- und Präbiotika (33-36). Bislang vorliegende Studien zeigen, dass das Darm-Mikrobiom von an RA und insbesondere an SpA Erkrankten different von Gesunden ist. Dies trifft insbesondere für frühe Stadien der Erkrankung und DMARD-naive Patienten zu. Es wird spekuliert, ob hier molekulare Ähnlichkeiten bzw Mimikry zwischen bakteriellen Komponenten und RA-assoziierten Antigenen zugrunde liegen. In metagenomischen Erhebungen zeigen RAPatienten vor allem mehr grampositive und weniger gramnegative Bakterien als Gesunde (37). Studien an Kindern mit SpA zeigen qualitativ ähnliche Veränderungen des Darm-Mikrobioms wie bei chronisch entzündlichen Darmerkrankungen (CED) (38). Gut belegt ist, dass bis zu einem Viertel der Patienten mit CED auch unter Arthritiden, meist SpA, leiden. Probiotische Präparate haben in die Behandlung chronisch entzündlicher Darmerkrankungen Eingang gefunden. Aus den vorliegenden
Fazit: Was kann empfohlen werden?

Als Basisernährung kann Patienten mit rheumatischen Erkrankungen eine mediterrane und möglichst pflanzenbasierte Ernährung ohne oder mit nur sehr wenig tierischen Produkten empfohlen werden. Auch sind polyphenolreiche Gemüse, Obst und Gewürze zu empfehlen. Darüber hinaus ist, sofern keine Kontraindikationen wie u.a. Essstörungen und Untergewicht vorliegen, ein Heilfasten ein- bis zweimal jährlich empfehlenswert, sofern es gut verträglich ist. Eine Fastentherapie sollte initial nur unter erfahrener ärztlicher Begleitung durchgeführt werden. Identifizieren Patienten Nahrungsmittel, die einen Schub wiederholt auszulösen scheinen, so kann eine Elimination empfohlen werden. Da Unverträglichkeiten, auch durch die Einflüsse des Mikrobioms, dynamischer Natur sind, sollten allerdings gelegentliche Re-Expositionen durchgeführt werden um langfristig unnötig einseitige Diäten zu vermeiden.

Assoziationen kann aber derzeit noch keine spezifische mikrobiologische Therapie bei rheumatischen Erkrankungen abgeleitet werden. In einer ersten kleinen randomisierten Studie führte die achtwöchige Gabe von Lactobacillus casei zu einer verringerten Krankheitsaktivität und Zytokinkonzentrationen bei Patienten mit RA (39). Die Ergebnisse weiterer Studien sind abzuwarten, bevor probiotische Präparate oder präbiotische Diäten empfohlen werden können.

\section{Interessenkonflikt}

Der Autor gibt an, dass kein Interessenkonflikt vorliegt.

\section{Literatur}

1. Kerekes G, Nurmohamed MT, González-Gay MA et al. Rheumatoid arthritis and metabolic syndrome. Nat Rev Rheumatol 2014; 10: 691-696.

2. Masuko KA. Potential Benefit of „Balanced Diet“ for Rheumatoid Arthritis. Front Med (Lausanne) 2018; 5: 141.
3. Darlington LG, Ramsey NW. Review of dietary therapy for rheumatoid arthritis. British journal of rheumatology 1993; 32: 507-514.

4. Sparks JA, Barbhaiya M, Tedeschi SK et al. Inflammatory dietary pattern and risk of developing rheumatoid arthritis in women. Clin Rheumatol 2018 Aug 14. [epub ahead of print]

5. Skoldstam L, Hagfors L, Johansson G. An experimental study of a Mediterranean diet intervention for patients with rheumatoid arthritis. Annals of the rheumatic diseases 2003; 62: 208-214.

6. McKellar G, Morrison E, McEntegart A et al. A pilot study of a Mediterranean-type diet intervention in female patients with rheumatoid arthritis living in areas of social deprivation in Glasgow. Annals of the rheumatic diseases 2007; 66: 1239-1243.

7. Tracy SW. Something new under the sun? The Mediterranean diet and cardiovascular health. The New England journal of medicine 2013; 368: 1274-1276.

8. Samaha F. Effects of a Mediterranean-style diet on cardiovascular risk factors. Annals of internal medicine 2007; 146: 73; author reply -4 .

9. Berkow SE, Barnard ND. Blood pressure regulation and vegetarian diets. Nutrition reviews 2005; 63: 1-8.

10. Fraser G, Katuli S, Anousheh R et al. Vegetarian diets and cardiovascular risk factors in black members of the Adventist Health Study-2. Public health nutrition 2015; 18: 537-545.

11. Kwok CS, Umar S, Myint PK et al. Vegetarian diet, Seventh Day Adventists and risk of cardiovascular mortality: a systematic review and meta-analysis. International journal of cardiology 2014; 176: 680-686.

12. Yokoyama Y, Nishimura K, Barnard ND et al. Vegetarian diets and blood pressure: a meta-analysis. JAMA internal medicine 2014; 174: 577-587.

13. Kjeldsen-Kragh J, Haugen M, Borchgrevink CF et al. Controlled trial of fasting and one-year vegetarian diet in rheumatoid arthritis. Lancet 1991; 338: 899-902.

14. Shim HS, Longo VD. A protein restriction-dependent sulfur code for longevity. Cell 2015; 160: $15-17$.

15. Longo VD, Mattson MP. Fasting: molecular mechanisms and clinical applications. Cell metabolism 2014; 19: 181-192.

16. Fontana L, Partridge L. Promoting health and longevity through diet: from model organisms to humans. Cell 2015; 161: 106-118.

17. Fontana L, Partridge L, Longo VD. Extending healthy life span - from yeast to humans. Science 2010; 328: 321-326.

18. Mattson MP. Energy intake, meal frequency, and health: a neurobiological perspective. Annual review of nutrition 2005; 25: 237-260.

19. Michalsen A, Li C. Fasting therapy for treating and preventing disease - current state of evidence. Forsch Komplementmed 2013; 20: 444-453.

20. Muller H, de Toledo FW, Resch KL. Fasting followed by vegetarian diet in patients with rheumatoid arthritis: a systematic review. Scandinavian journal of rheumatology 2001; 30: 1-10.

21. Hafstrom I, Ringertz B, Spangberg A et al. A vegan diet free of gluten improves the signs and symptoms of rheumatoid arthritis: the effects on arthritis correlate with a reduction in antibodies to food 
antigens. Rheumatology (Oxford) 2001; 40: $1175-1179$

22. Al-Okbi SY. Nutraceuticals of anti-inflammatory activity as complementary therapy for rheumatoid arthritis. Toxicology and industrial health 2014; 30: 738-749.

23. Ramadan G, Al-Kahtani MA, El-Sayed WM. Antiinflammatory and anti-oxidant properties of Curcuma longa (turmeric) versus Zingiber officinale (ginger) rhizomes in rat adjuvant-induced arthritis. Inflammation 2011; 34: 291-301.

24. Ramadan G, El-Menshawy O. Protective effects of ginger-turmeric rhizomes mixture on joint inflammation, atherogenesis, kidney dysfunction and other complications in a rat model of human rheumatoid arthritis. International journal of rheumatic diseases 2013; 16: 219-229.

25. Anand R, Kaithwas G. Anti-inflammatory potential of alpha-linolenic acid mediated through selective COX inhibition: computational and experimental data. Inflammation 2014; 37: 1297-1306.

26. Riegsecker S, Wiczynski D, Kaplan MJ, Ahmed S. Potential benefits of green tea polyphenol EGCG in the prevention and treatment of vascular inflammation in rheumatoid arthritis. Life sciences 2013; 93: 307-312.
27. Roy S, Sannigrahi S, Vaddepalli RP et al. A novel combination of methotrexate and epigallocatechin attenuates the overexpression of pro-inflammatory cartilage cytokines and modulates antioxidant status in adjuvant arthritic rats. Inflammation 2012; 35: 1435-1447.

28. Shukla M, Gupta K, Rasheed Z et al. Consumption of hydrolyzable tannins-rich pomegranate extract suppresses inflammation and joint damage in rheumatoid arthritis. Nutrition 2008; 24: 733-743.

29. Singh S, Nair V, Gupta YK. Linseed oil: an investigation of its antiarthritic activity in experimental models. Phytotherapy research 2012; 26: 246-252.

30. Cameron M, Chrubasik S. Oral herbal therapies for treating osteoarthritis. The Cochrane database of systematic reviews 2014; 5: CD002947.

31. Chandran B, Goel A. A randomized, pilot study to assess the efficacy and safety of curcumin in patients with active rheumatoid arthritis. Phytother Res 2012: 1719-1725.

32. Amalraj A, Varma K, Jacob J et al. Novel Highly Bioavailable Curcumin Formulation Improves Symptoms and Diagnostic Indicators in Rheumatoid Arthritis Patients: A Randomized, DoubleBlind, Placebo-Controlled, Two-Dose, Three-
Arm, and Parallel-Group Study. J Med Food 2017; 20: $1022-1030$

33. McLean MH, Dieguez D Jr., Miller LM, Young HA. Does the microbiota play a role in the pathogenesis of autoimmune diseases? Gut 2015; 64: 332-341.

34. Scher JU, Abramson SB. The microbiome and rheumatoid arthritis. Nature reviews Rheumatology 2011; 7: 569-578.

35. Taneja V. Arthritis susceptibility and the gut microbiome. FEBS letters 2014; 588: 4244-4249.

36. Yeoh N, Burton JP, Suppiah P et al. The role of the microbiome in rheumatic diseases. Current rheumatology reports 2013; 15: 314.

37. Zhang $\mathrm{X}$, Zhang $\mathrm{D}$, Jia $\mathrm{H}$ et al. The oral and gut microbiomes are perturbed in rheumatoid arthritis and partly normalized after treatment. Nat Med 2015; 21: 895-905.

38. Stoll ML, Cron RQ. the microbiota in pediaric rheumatic disease:epiphenomen or therapeutic target ? Curr Opin Rheumatol 2016; 28: 537-543.

39. Vaghef-Mehrabany E, Alipour B, Homayouni-Rad A et al. Probiotic supplementation improves inflammatory status in patients with rheumatoid arthritis. Nutrition 2014; 30: 430-435. 